International Journal of Advanced Information Technology (IJAIT) Vol. 1, No. 3, June 2011

\title{
INFORMATION MODELING AND KNOWLEDGE MANAGEMENT APPROACH TO RECONFIGURING MANUFACTURING ENTERPRISES
}

\author{
Muhammad Baqar Raza, Robert Harrison \\ Wolfson School of Mechanical and Manufacturing Engineering \\ Loughborough University, Loughborough, LE11 3TU, UK. \\ mmbrmelboro. ac $\cdot u k$
}

\begin{abstract}
This research aims to gain a detailed understanding of data producers, data consumers and format/flow of the data within automotive industry for defining and using Bill of Process (BoP) for engine assembly lines. The focus remained on the real industrial challenge of rapid constraint evaluation for designing and/or reconfiguration of Powertrain assembly lines to cater for a new/changed product. A methodology is developed to facilitate Ford Company to quickly model and re-configure new/changed assembly line for building new/changed engine. This is made possible with the help of modular approach and developing relationships among products, processes and resources. The data and information of the PPR is made available to all the stake holders of the organisation independent of the platform or specific application being used at the department/facility.
\end{abstract}

\section{KEYWORDS}

Modularity, Re-configurability, Ontology, Knowledge based system, knowledge management.

\section{INTRODUCTION}

The recent advancements in the information and telecommunications have made possible global alliances among enterprises while changing customer needs are characterised by a rapidly emerging global market economy. Customisation, performance, quality and environmental issues are becoming predominantly the differentiators in defining the success of the manufacturing industries in terms of increased revenue and profitability. Products entering into the markets are designed and manufactured across geographical boundaries, distributed and marketed worldwide. In addition to the worldwide challenge, the companies are also facing many challenges such as shorter time to market, improved process yield, non-stop operations, tighter supply couplings, newer and innovative technologies, complexities in products and larger/virtual organisations.

Product Lifecycle Management (PLM) system is a sequence of steps including product design and development process that enterprises employ to conceive design and commercialise the product. The manufacturing efficiency and competitive advantage can be improved when enterprises capitalising on an entire product lifecycle chain to bring real time shop floor visibility across the enterprise. Furthermore, faster time to market can be achieved when manufacturing requirements are synchronised with the product design and geographically

DOI : 10.5121/ijait.2011.1301 
distributed manufacturing operations to meet the global market demands. Lifecycle support helps the manufacturers to bring more innovative products to market faster, leveraging the power of global manufacturing operations, improving production efficiency, maintaining quality and boosting profitability. However the lifecycle support is incomplete without the suppliers' involvement to bring more competitiveness in today's manufacturing environment. Therefore, manufacturers demand the best in class solutions from a variety of vendors and partners.

The automobile industry has provided revolutionary manufacturing management paradigms throughout the century. Mass Production System of Ford (MPS) and Lean Production of Toyota called Toyota Production System (TPS) successfully flourished during last century in manufacturing and especially automotive industry. The beginning of this century has introduced Modular and Re-configurable Strategies to the manufacturing enterprises. The overall objectives and scope of this research work originate in the interest in exploring the applicability and appropriateness of adapting the fledgling concept of re-configurability of automation systems based upon modular platform and applying these adapted methods to real world problems.

\section{Challenges AND OPPORTUNities}

There is no denying the fact that the automotive sector is under a continuous pressure of many factors such as globalisation, customisation, performance, quality and environmental issues etc. The product's life has reduced from years to months. The continuity in change is because of several reasons including customer changed requirements, necessity to variety, technology advancement, changing environmental regulations, increase safety issues and many more. Life of the assembly machines and resources surpass to the life of the products made out of them. Heavy investment can go unutilised or wasted when the product changes. Every time there is a change in the product, it is associated with heavy costs and cumbersome task of changing the system.

Current automation systems fail to meet business requirements. The assembly lines, such as powertrain assembly line for automotive engine, have a limited capacity to produce variety of products (engines). The built in capability to deal with variety has to be limited to justify investment and is a trade-off between the inevitable but unpredictable changes and the increased cost of flexibility. One of the main reasons for automation systems and especially the assembly automation systems in automotive sector not fulfilling the business requirements is that the relational knowledge base among products, processes and resources is either non-existent or not properly designed/used.

New strategies are urgently required to ensure faster commissioning and ramp-up through reconfiguration and rapid response to the unpredictable changes to enable manufacturers to become more competitive. The goal is the reduction in manufacturing lead-times and production ramp-up time i.e. the time to prepare production lines for different products. The current level of automation system is not appropriate when viewed in flexible business context of today's manufacturing systems. This may be improved through: (a) the provision and use of pre-build modules to achieve reconfigurable manufacturing systems which are also easily scalable as product changes, and (b) generation of dependency relationships among products, processes and resources to help evaluate the effects of changed products automatically through addition, removal or re-configuration of the existing systems in a rapid and cost effective manner.

The relationships among the product, process and resource (PPR) are not readily available in a form for decision making purposes. There is a genuine requirement from industry to establish relationships among PPR domains to readily assimilate the requisite information for any change in product at any time. The development of the dependency relationship among PPR is not easy because PPR domains are usually dealt by separate teams and are considered independent 
activities. This necessitates generating a cross-functional knowledge base which includes knowledge about products, processes and resources in the form of parameters, properties and arguments and a mechanism to link the dependent parameters automatically. The solution is described pictorially in figure 1 where product, process and resource information is integrated to lay down the basis of Ontological Knowledge Based System (OKBS) which in turn is the basis of Modular, Reconfigurable and Business Driven Automation (MRBDA) systems.

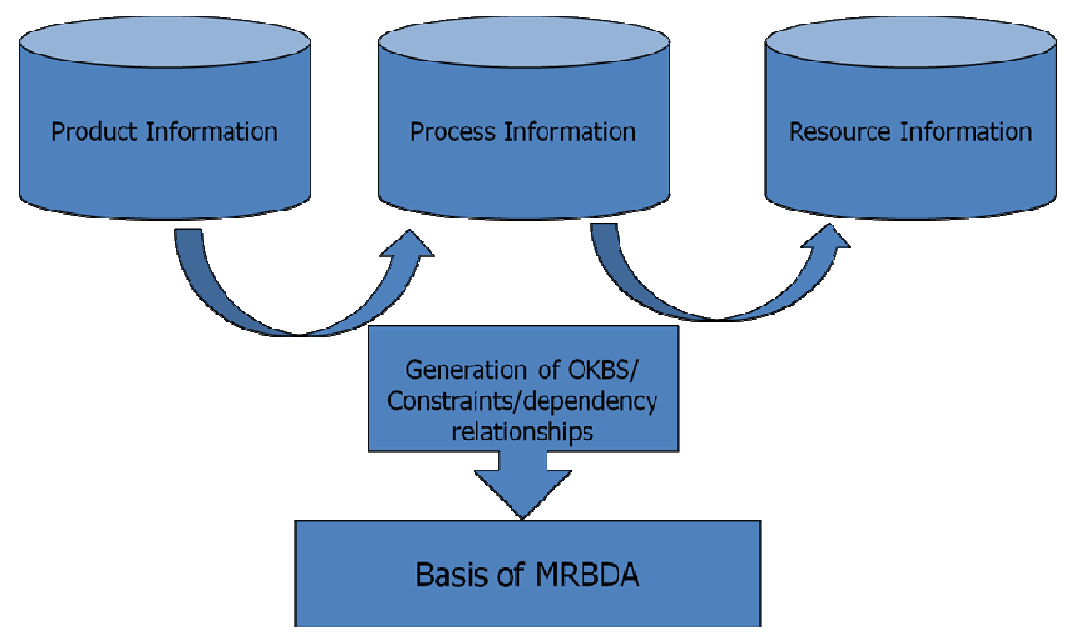

Figure 1. PPR Integration through ontology

\subsection{Enterprise Integration}

Enterprise Integration is the breaking down organisational barriers to improve synergy within enterprise so that business goals can be achieved in a significantly more productive and efficient way [1]. Globalisation brings new economic trends and is forcing companies to change their strategic vision. To remain competitive and reactive enterprise networks are required to adjust constantly their objectives with fluctuating market conditions. Enterprise integration is one way to achieve this goal. Enterprise Integration (EI) is deployed where there is a need in improving the task level interactions among people, systems, departments, services and companies [2]. A broader definition of EI is proposed by Li and Williams [3] stating that, "enterprise integration is the coordination of all elements including business, processes, people and technology of the enterprises working together in order to achieve the optimal fulfilment of the business missions of that enterprise as defined by the management". Three levels of integration within enterprise are addressed by Vernadat [2] and Li and Williams [3] i.e. (i) physical system integration, (ii) application integration and (iii) business integration.

\subsection{Product, Process \& Resource (PPR) Relationships}

Products, processes and resources have direct relationships with each other. Only those products can be manufactured cost effectively for which a resource supported process is present. Process selection has a very close relationship with the production volume. Same process may be used in a job shop and the mass production environment but the machines used to build the product will be different or the same machines may be used but the process might be different. 


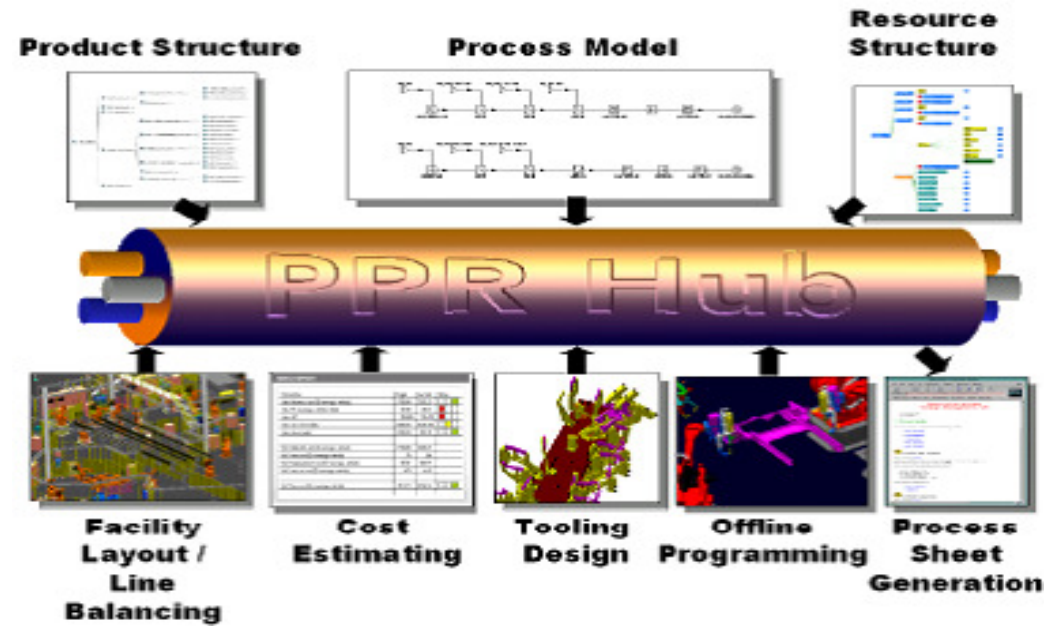

Figure 2. The use of PPR Hub (Adopted from Delmia Automation)

The figure 2 depicts the core research motive regarding the relationship between product, process and resources. There is a need of a knowledge base or a hub (Delmia terminology) containing integrated engineering information regarding products, processes and resources and available to all the stake holders which could be used when and where required. This knowledge base or hub should be utilised from the very beginning of the product design till finalisation of manufacturing/prototype to the point when there is a change in the product. The PPR hub represents products, processes and resources which are interdependent but there is no mechanism to show the interdependency or relationship among them. This is one of the research motivations which is lacking in the prevalent state-of-the-art automation systems.

\section{LITERATURE SURVEY}

Choosing and applying the best philosophies and techniques is not easy as most manufacturing enterprises (MEs) deploy complex and unique configurations of processes and resource systems and seek economies of scope and scale in respect of changing and distinctive product flows. It follows that systematic methods of achieving model driven reconfiguration and interoperation of component based manufacturing systems are required to design, engineer and change future MEs.

\subsection{Modularity and Modular Manufacturing}

A substantial literature stream suggests that many products are becoming more modular over time, and that this development is often associated with a change in industry structure towards higher degrees of specialization. Research on modularity and commonality has grown substantially over the past few years. Mostly the research focuses on modularity of products and a few on the processes as well. The subjects of these studies have been products, processes, organizations, and even innovations, although the set of references shows a strong preference towards products. Despite the wealth of existing research, there are still significant opportunities for future research [4].

A modular machine can be defined as a machine constructed from a set of standardised modules. The set of standardised modules allow constructing a wide variety of machines from a set of standardised components. Modular systems can easily be upgraded as better methods for assembly operations are developed as each module could be replaced independently. Finally 
modular system will be more robust to failure. If one module is failed, it will easily be identified and replaced

\subsubsection{Product Modularisation}

Product is composed of autonomous units or modules that are manufactured as sub-assemblies and then assembled together. Modularisation, to create a product composed of modules, provides product flexibility by means of combining developed modules together in various ways to extend the product range [5].

\subsubsection{Process Modularisation}

By implementing a modular strategy, product and process will naturally become more interlinked by providing a stable and common platform to design and manufacture. Modularity should thus increase the robustness and flexibility of a product and its associated manufacturing process/system. An extension to the theory of modularity for product design relates the module concept to processes and also to businesses. In addition to a system that will be able to integrate changes much more easily and rapidly throughout a products lifecycle.

\subsubsection{Resource Modularisation}

Selecting modular approach to assembly led to the development of two high level system concepts

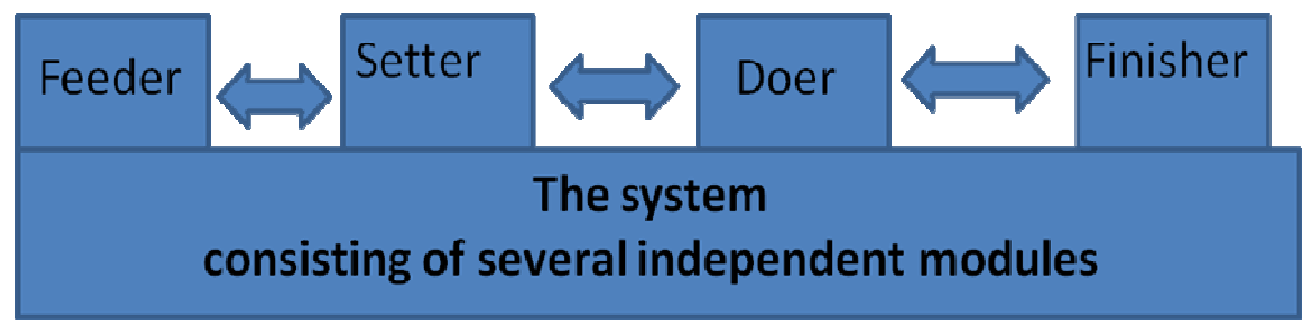

Figure 3. Hypothetical example of a modular machine system

The figure 3 shows the concept of modules for any product like a general machine. The machine can be broken down into functionally independent modules which make it a modular machine. Any module can be replaced if required with the new one keeping the machine running all the time. In addition to this, the Doer module, for example, may be replaced with another function and the same machine can be used for a different operation for the same family of machines.

\subsection{Flexible Manufacturing}

Flexibility is defined as the ability to change over to produce multiple products within a family quickly and economically. Shorter product lifecycles driven by technological innovation and intense competition has made the flexibility of a manufacturing system a valuable source of competitive advantage [6].

Chryssolouris [7] has classified the flexibility of manufacturing system as:

- Machine Flexibility: The ease of making changes required to produce a given set of part types

- Process Flexibility: Ability to produce a given set of part types, possibly using different materials in different ways 
- Product Flexibility: Ability to change over to produce new (set of) products very economically and quickly

- Routing Flexibility: Ability to handle breakdowns and to continue producing a given set of part types

- Volume Flexibility: Ability to operate profitably at different production volumes

- Expansion Flexibility: Ability to expand the system easily and in modular fashion

- Operation Flexibility: Ability to interchange ordering of several operations for each part type

- Production Flexibility: The universe of part types that the manufacturing system can produce

Automotive companies are disappointed by FMS with past experience for machining applications because the cost reduction promise for equipment reuse has not been materialised as expected [8]. In practice, to respond to quickly changing market demands, FMS requires significant additional expenditures and a long time to convert or adapt a new unplanned product. Thus FMS do not meet agility criteria i.e. rapid and cost effective reuse in response to changing product demands [8].

\subsection{Re-configurability}

Re-configurability is defined as the ability to repeatedly change and rearrange the components of a system in a cost-effective way which is being extensively used in computing, automated assembly and robotics. Then, the evolution of manufacturing, from dedicated to flexible manufacturing systems, is briefly discussed and the need for reconfigurable manufacturing systems is outlined. It is shown that there are common research issues in reconfigurable computing, robotics and manufacturing such as system-module-component interfaces, design methodologies, modularity, tools and tool suites development, strategic analysis and business modelling, training, and support.

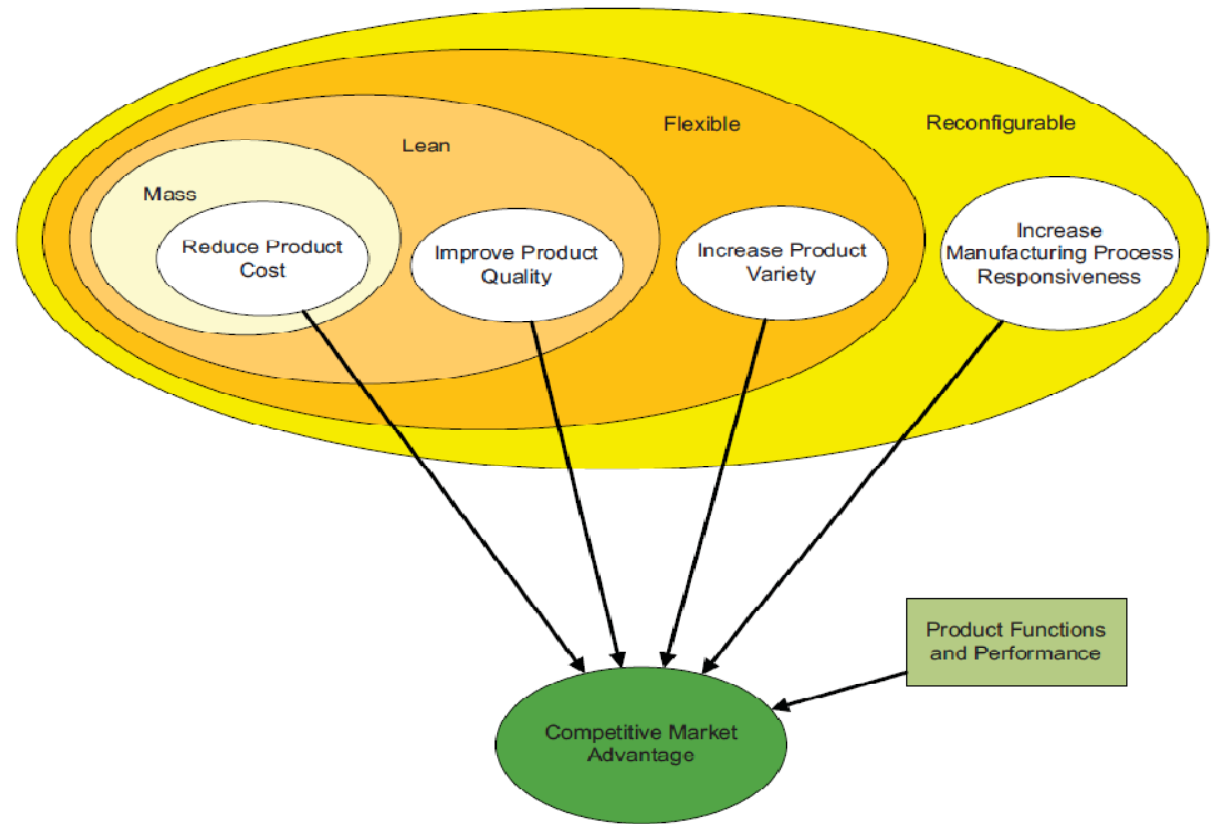

Figure 4. Economic goals of various manufacturing paradigms [9]

A re-configurable manufacturing system (RMS) is designed for rapid adjustment of production capacity and functionality in response to new market conditions and new process technology as 
shown in figure 4. It has several distinct characteristics including modularity, integrability, customisation, convertibility and diagnosability. There are a number of key interrelated technologies that should be developed and implemented to achieve these characteristics.

Re-configurability, an engineering technology that deals with cost-effective, quick reaction to market changes, is needed. Reconfigurable manufacturing systems (RMS), whose components are reconfigurable machines and reconfigurable controllers, as well as methodologies for their systematic design and rapid ramp-up, are the cornerstones of this new manufacturing paradigm. A RMS is designed for rapid adjustment of production capacity and functionality in response to new market conditions and new process technology. It has several distinct characteristics including modularity, integrability, customisation, convertibility and diagnosability.

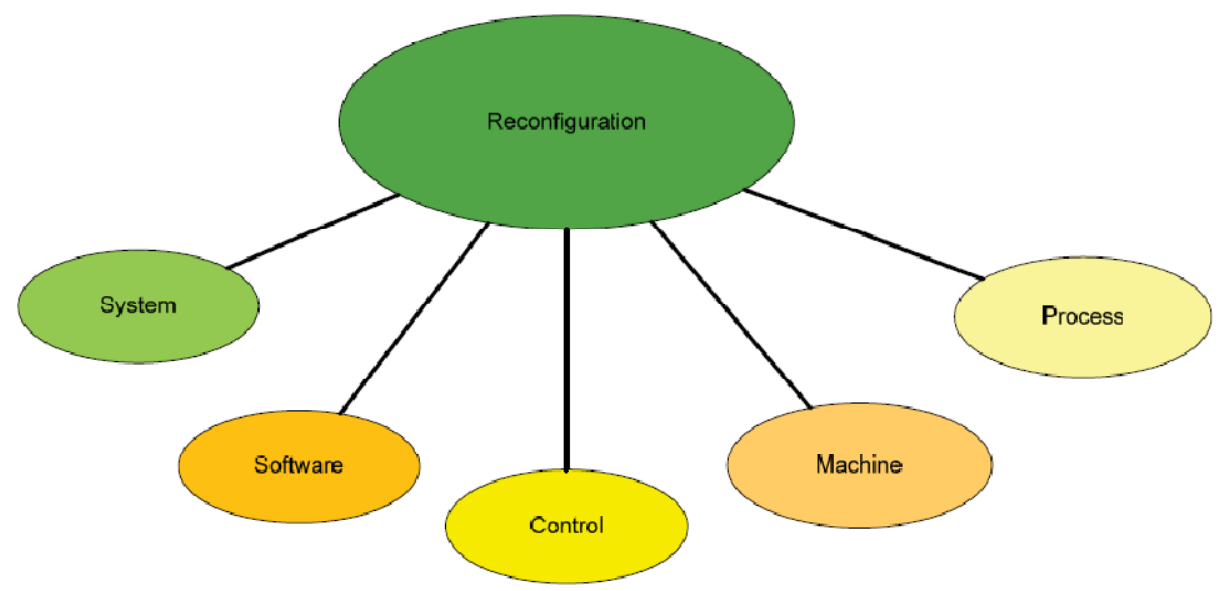

Figure 5 Aspects of reconfigurable manufacturing systems [9]

Re-configurability, in general, is a very large topic and deals with many domains as depicted in figure 5. The scope of this work cannot cover all kinds of re-configurable systems. In this case the automotive industry provides the context for the kind of re-configurability system to be focused. This kind of re-configurability system can be characterized as the development of automation system for the assembly of high variety of products based upon modular platform. The importance of information and knowledge sharing in manufacturing sector of automotive industry is beyond any argument. Knowledge sharing and management is pervasive for success of any manufacturing paradigm. Similarly re-configurability based upon modularity is no exception to this. This research explores ways and methods to share and manage information and knowledge for better implementation of the philosophy.

\subsection{Agile Manufacturing}

Agile manufacturing is the ability to adapt quickly and profitably to continuous and unexpected changes in the manufacturing environment. The impact of agility is on the entire manufacturing enterprise including design, customer relations, logistics as well as production. Therefore with agility manufacturing emerged from an era of mass manufacturing to mass customisation and companies are bound to use new technologies and manufacturing concepts [10].

\section{SMART ASSEMBLY}

The Manufacturing Engineering Laboratory (MEL) of the National Institute of Standards and Technology (NIST) has been working on the concept, design and implementation of smart assembly systems. The definition as described by NIST's MEL is: "The vision for Smart Assembly is a system consisting of the optimal balance of people and automation interacting 
effectively, efficiently, and safely. People work in knowledgeable, empowered work teams that utilize best assembly practices and technologies. Virtual optimization and validation of assembly processes are used to ensure the best designs work the first time. Effective integration of automation and information technology into the human assembly process maximizes total system performance on a consistent basis. And finally, flawless execution of supply chain and product life cycle processes successfully synchronizes the entire assembly".

\subsection{Key characteristics of Smart Assembly Systems}

- Empowered, knowledgeable people: A multi-disciplined, highly skilled workforce is empowered to make the best overall decisions.

- Collaboration: People and automation working in a safe, shared environment for all tasks.

- Reconfigurable: Modular "plug and play" system components are easily reconfigured and reprogrammed to accommodate new product, equipment, and software variations, and to implement corrective actions.

- Model and Data Driven: Modeling and simulation tools enable all designs, design changes, and corrective actions to be virtually evaluated, optimized, and validated before they are propagated to the plant floor. The "virtual" models and real-time plant floor systems are synchronized.

- Capable of Learning: Self integrating and adaptive assembly systems prevent repeated mistakes and avoid new ones.

Smart Assembly is the incorporation of learning, reconfigurability, human-machine collaboration, and model-based techniques into assembly systems to improve productivity, cost, flexibility, responsiveness and quality.

\subsection{Enabling Technologies for Smart Assembly}

Manufacturing has become information and knowledge intensive, requiring the sharing of information accurately, inexpensively, and seamlessly throughout the extended enterprise and supply chain.

1) Intelligent flexible assembly processes, equipment and tools

2) Accurate, easy-to-use, pervasive, persistent, virtual capability

3) Effective plant floor decision support tools, actionable information for man and machine i.e. interoperability from "shop floor" to "top floor" [11].

General Motors (GM), in collaboration with the National Institute of Standards and Technology's (NIST) Manufacturing Engineering Laboratory (MEL), is developing a broad industry definition and vision for Smart Assembly, and is beginning to develop the technology and business process "roadmaps" that will provide a framework to focus and prioritize both current and future research and development in this area [11]. Similar efforts have been under development and implementation at Ford's Dunton Technical Centre (DTC), Ford Motor Company, UK in collaboration with Loughborough University, UK.

\section{KNOWLEDGE MANAGEMENT}

Knowledge is defined as application of the (available) information. Hopgood [12] describes that fundamental aim of the research in AI is to build a machine that can think, mimic or even exceed human mental capabilities. However this aim is far from achieved and intelligent systems built today just mimic human capabilities in certain area such as logic reasoning. According to Hopgood [12], intelligence is the capacity to acquire and apply knowledge while knowledge is referred to as understanding and awareness which are normal human capabilities. 
Therefore in AI terms, a machine is not intelligent if it cannot learn outside its programmed boundaries still it can be called intelligent when performing tasks as done by humans. Hopgood [12] has defined intelligent systems to be of 03 main types: (i) knowledge based system, (ii) computational Intelligence and (iii) hybrid systems. The focus of the research is purely on knowledge based systems

\subsection{Knowledge Based Systems}

Knowledge based systems or knowledge based engineering systems distinguish themselves from conventional systems by not storing the used domain knowledge intertwined with instructions for controlling the application of this knowledge. In other words knowledge base is separated from its controlled mechanism called inference engine as shown in figure 6, [12], [13].

Knowledge based systems are classified as expert system, rule base systems, frame based systems or intelligent agents [12]

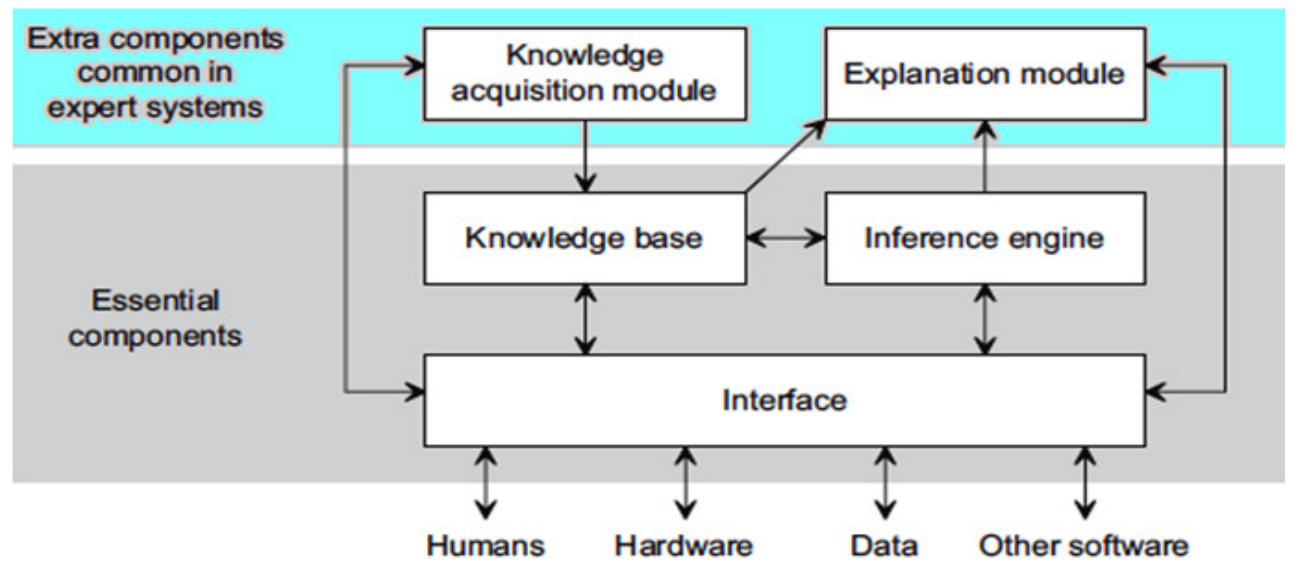

Figure 6. Main components of knowledge based systems [12]

Ontologies are not only useful for helping solving the information overload problem, but can be used for a variety of different applications, such as sharing explicit knowledge, increase communication, and help in natural language understanding. Knowledge Management can be broken into four stages that require different activities. (i) Knowledge Capture is the identification of structure and the collection of data into that structure. This might occur in special activities aimed at developing structure or organizing data, or it might happen implicitly during the accomplishment of an activity. The result is a data store with an organization that is of greater value than the isolated facts. (ii) Knowledge Representation is defined as a formal rigorous knowledge representation from different information sources in the form of a reference architecture, ontology provides such reference architecture. (iii) Knowledge Access is the set of methods for inquiring of or investigating the knowledge store. The knowledge store organization can be complex and finding information, drawing conclusions or identifying patterns requires mechanisms for finding, collecting, aggregating and displaying desired information. (iv) Knowledge Application is the end act of using the knowledge found in the store in a current activity. This is the purpose of Knowledge Management and the reason for collecting the information, maintaining the store, and studying its contents.

Ford is using Teamcenter (TC) and e2ks software applications for capturing, reuse and management of knowledge. In the initial phase of the research it was planned to use the existing 
technology to develop PPR dependency relations but soon it was realised that the available technology is not sufficient for the intended purpose. E2ks supports decision making especially related to manufacturing of products and not for assembly of products while TC is designed for information management and not for knowledge management.

\subsection{Ontology - Foundation of a knowledge base}

The subject of ontology is the study of the categories of things that exist or may exist in some domain. Ontologies in combination with logical formalisms provide a 'semantical-logical' language in which logical relationships between objects can be expressed. This introduces the possibility to perform reasoning with objects and makes the ontology a tool for generating new objects e.g. automated reasoning with transitive and reflexive logical properties of classes can identify inconsistencies or gaps in the ontology. In addition, automated reasoning can be used to constrain domain specific semantics of possible future facts.

To implement the proposed 'To-be' system, it is required to use an open, scalable, and unified knowledge base to act as a single system; it is proposed to use ontologies as enabling technology. An ontology is a machine understandable model of the domain of interest which contains all the entities, their properties, relationships, categorisation as well as constraints, rules etc. It may be called an intelligent database which understands its domain which forms the basis of communication between people and among machines. Ontology is used to share and standardise common understanding of the structure of information and to enable storage, capture and reuse of domain knowledge that previously was limited to individual engineer expertise. Ontology domain and scope is very important for successful results. The ontology would be linked to CCE and would be specifically used for designing / redesigning / reconfiguration of engine assembly lines.

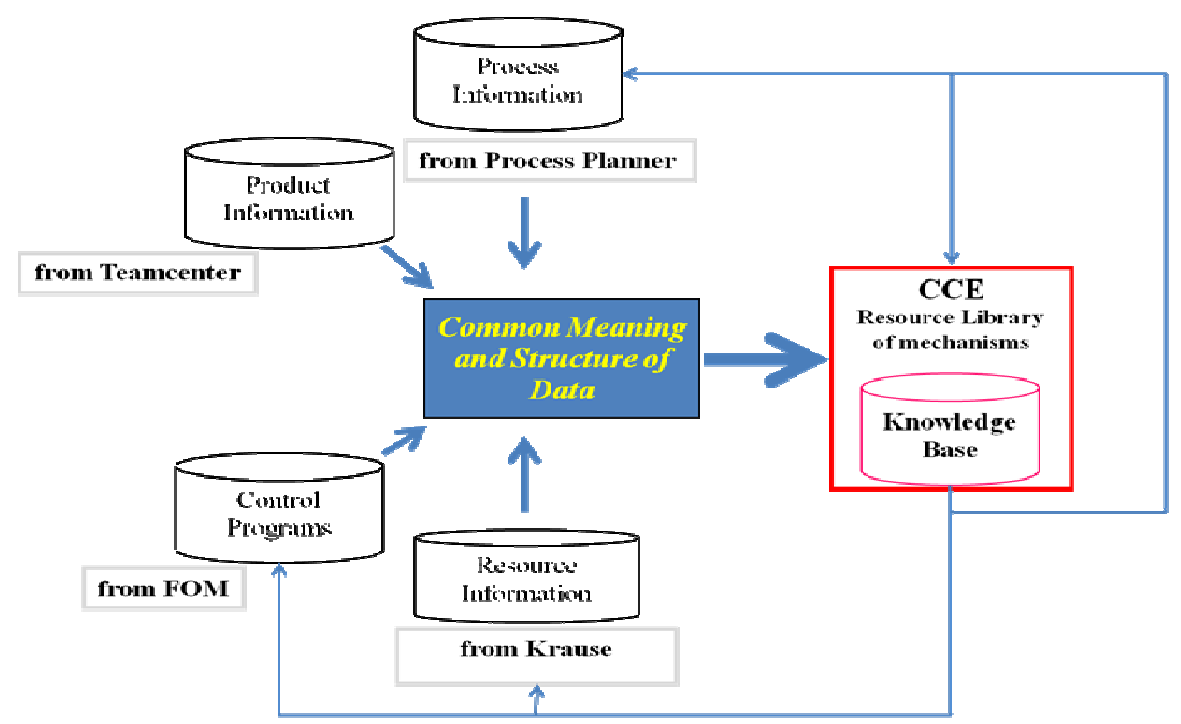

Figure 7. Ontological support in legacy applications to design KB system

\subsection{Ontology Languages - WSML vs. OWL}

OWL is $\mathrm{W} 3 \mathrm{C}$ recommended ontology language. The semantic web initiative conceived and led by the $\mathrm{W} 3 \mathrm{C}$ yielded the ontologies as one of the solutions for its implementation. OWL is developed for web use, its utilisation enables the required interoperability. In addition to this, its inherent reasoning capability is the other characteristic which has been achieved with this language. Expressivity and reasoning capabilities are important requirements at the moment of choosing an ontology language, OWL offers both these characteristics. 
In the current project, Web Services Modeling Language (WSML) is used to build ontologies which is relatively a new language based on logic-based knowledge representation formalisms, namely Description Logics and Logic Programming [14]. It specifies XML and RDF serialisations to be compatible with existing web standards. The WSML syntax is split into two parts: (i) the conceptual syntax, and (ii) logical expression syntax. The general logical expression syntax for WSML has a first-order logic style. Additionally, WSML provides extensions based on 'F-Logic' as well as 'Logic Programming' rules and database-style integrity constraints [14].

\subsection{PPR Ontology}

The manufacturing sector is currently presenting urgent needs to swiftly accommodate new products, rapidly establishing new factories or retrofitting existing ones. In order to achieve this goal, engineering tasks which are currently performed manually need to be automated. In this scenario, ontologies emerge as a sound solution to represent knowledge on manufacturing processes, equipment and products in a machine interpretable way. This knowledge then can be used by automated problem solving methods to (re)configure the control software that coordinates and supervises manufacturing systems. The use of ontologies in manufacturing domain has evolved over the last decade. Recently ontologies began to find a place in manufacturing automation architectures as a part of bigger efforts focussing on multi-agent systems for manufacturing control. Ontologies provide a solution for knowledge representation, inference and present a language human understandable and machine interpretable.
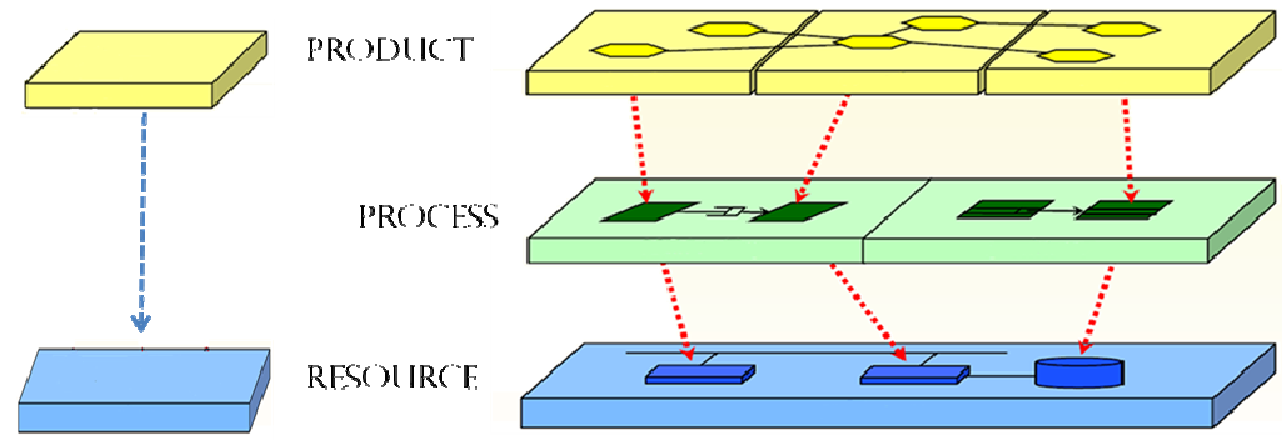

Figure 8. Products are related to resources through processes

Ontologies provide a solution to overcome current limitations presented by the technical specifications of the products, by the processes required to manufacture them and by devices and machines used, which are written in natural language often aided by visual diagrams. Relations between product and resource are established through processes as shown in figure 8 . These relations are combined by pre-existing know-how and experience of engineers to systematically develop the layout of the factory and the automatic control programs which coordinate and supervise the processes. Whenever changes are needed to accommodate a new product or process, engineers are brought in to match the specifications established by the product design and system configurations. 


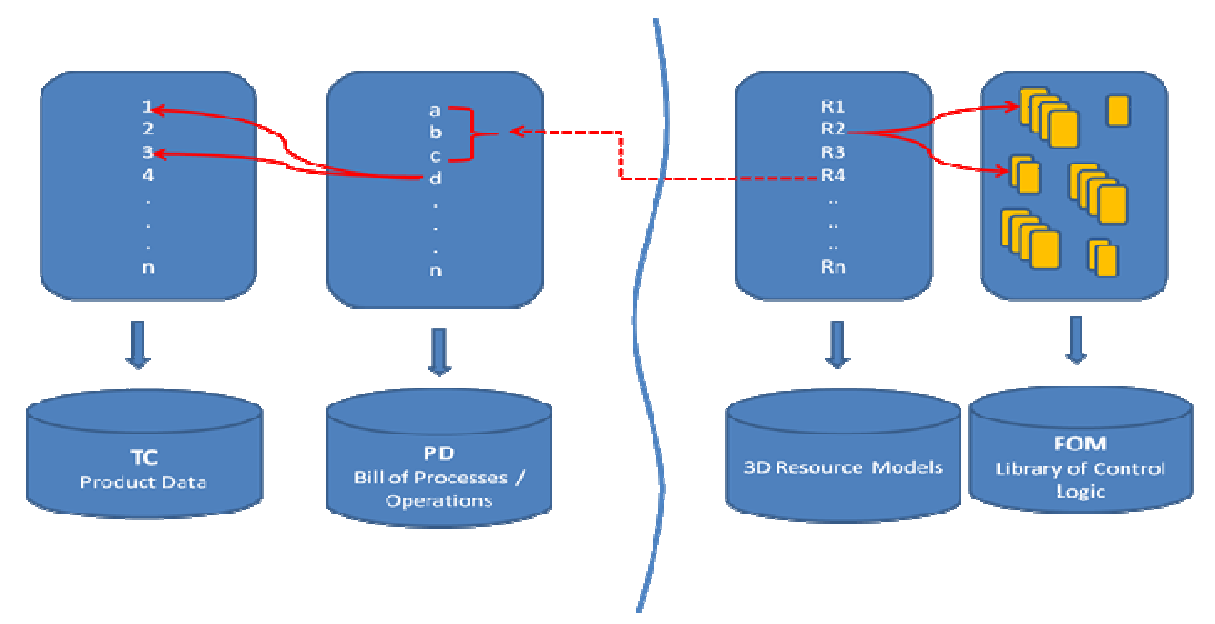

Figure 9. Current state of disparate applications

With the help of the CCE tool, the modular architecture is introduced in the designing of the assembly machines. As shown in figure 9, CCE, TC, PD and FOM are standalone (software entities that are defined in isolation and are operated autonomously), heterogeneous (refers to the fact that each application system implements its own data model defining its relevant concepts in its own way), autonomous (means that application systems update their internal instance data independent of any other application systems) and distributed (means that applications systems in general implement their data model in their own data repository and these are not shared with other application systems) applications.

A prototype is constructed for supporting ontology mapping and data sharing within Ford company, where users can collaborate on aligning their ontologies, and manually-driven alignments can be stored and reused later. The alignments can then be exported or used to translate queries into different ontologies for merging results from different data sources. Services for handling the discovery and exploitation of instance mappings would also be developed. Such service will be of paramount importance for the data integration task since the information relative to the same topic is scattered in different data sets with Ford as well as across its supply chain partners.
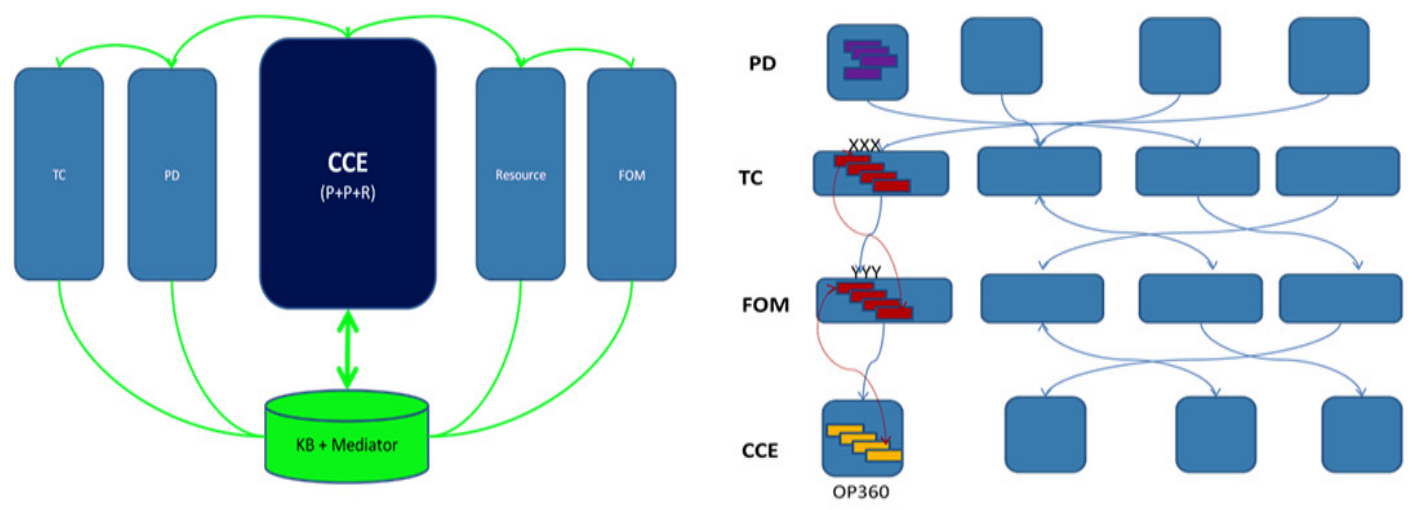

Figure 10. Ontology application - matching components through mapping

The mapped concepts within the relevant application domains at Ford i.e. Process Designer (PD), Teamcenter (TC), Function Oriented Modularity (FOM), and Core Component Editor 
(CCE), can be accessed through ontologies as shown in figure 10. Ontologies formally specify the terminology used to describe functionalities and behaviour. Mediators are used to allow interoperability between heterogeneously described concepts / sub-concepts, a particular type of mediator being the data mediator. A data mediator is used to solve terminological mismatches that arise when two different ontologies are used to describe two concepts / services. When a concept / service interact with another, the queries and the resulting instance data must be translated from terms of service into terms of the other. These processes are known as query rewriting and instance transformation. This is implemented in the prototype at Ford. The applications used in the re-configuration process are all mediated by data mediator and instances can be transformed from one ontology to the other. It is intended to address the problem of specifying transformation of the instances as part of the mapping specification between two ontologies.

\section{RESEARCH AT FORD}

Ford Motor Company in UK is involved in research, development, implementation and production of vehicle assembly operations. One of the most critical areas is powertrain (engine) assembly. Ford in UK is responsible for assembly machines' development, production and defining assembly sequence, productivity issues etc. Assembly machines are designed, built and commissioned by supply chain partners e.g. Krause which is a geographically distant vendor based in Germany. Machine building and especially commissioning takes a huge amount of time which in turn delays the overall program targets. There are several reasons for these delays. New product development or any variant of a product in production is an iterative activity of constraints evaluation and approval for production from various perspectives involving several stake holders. Once the product is released to machine builders, it may still be undergoing minor changes which slow down the process of machine building. Sometimes machine builders may suggest a few changes in product to make it cost effective. Also the virtual verification of the machines is carried out almost independently by the machine builders. Machine builders analyse and verify assembly machines from just the operational point of view of the resources (machines) only, leaving bigger picture of assembly sequence, presence of other machines and plant layout behind. This virtual verification of the whole assembly line is carried out by Ford using Process Designer (PD) software. PD is a heavy weight application and takes huge time to simulate the virtual commissioning therefore it is usually restricted to suspected workstations only. Thus it becomes a complex collaboration activity where responsibilities fluctuate between Ford and its vendors.

In addition to this, the relational knowledge base among products, processes, operations and resources is limited and tacit. Whenever there is any change in the product, this forces process engineers to re-design / re-configure new or existing machines with the previous knowledge of the 'product vs. resources' parameters implicitly. The change in operational parameters is manually checked as there is no information how the change in product could affect corresponding changes in the machines. Once the initial process of changes in the machines is outlined, different data formats again hinder to effectively manage changes across supply chain vendors.

The whole idea is to establish relationship between PPR in an organised and logical way and make this knowledge available to all the stake holders throughout the company and across supply chain partners. For this, the ontological representation of stations on one of the zones of the engine assembly line is carried out. Stations of the engine assembly line is decomposed to basic building blocks i.e. modules of mechanisms which are independent to each other and can perform one assembly operation independently. 

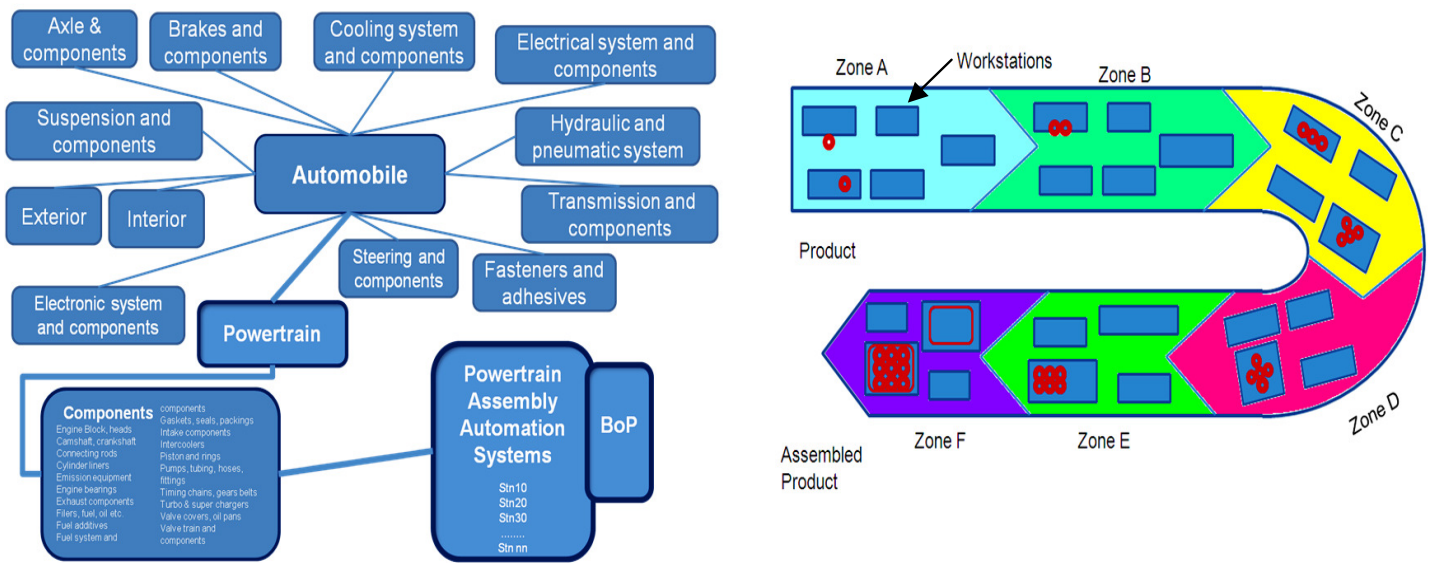

Figure 11. Automobile systems and conceptual diagram of powertrain assembly line

Engine assembly line is a highly sophisticated and complex combination of sequential operations and activities which are mostly automatic as well as automobile itself is a complex system. A schematic detail of the simplified description of assembly line is provided in figure 11. Engine assembly is a multifarious engineering activity involving several software applications and diverse engineering teams. Presently, the data available at Ford is not homogeneous and different formats are used at different departments/facilities. This is not supported by all the software applications being used which restrict it to be used across all the departments. No relationships among PPR have been established as yet and the dependency constraints among PPR are tacit and based upon human judgement.

\section{IMPLEMENTATION}

The aim of this case study is to provide an overview of the work carried out by Loughborough University and Ford Motor Company, UK under the Business Driven Automation (BDA) [15] project as well as to gain an understanding and possible improvement in designing / reconfiguration of assembly machines and the format/flow of the data within this process. By introducing the standardised (i) library modules of machines, (ii) data structures and (iii) ontological knowledge management techniques in legacy software applications and Core Component Editor (CCE) tool, a revised and improved business case is presented here.

\subsection{Justification of the KB System with Modular Re-configurability Tool}

To automate (fairly) the task of assembly line configuration / reconfiguration, product and resource (line) link points need to be defined at early stages of design and made available easily to be searched, analysed and implemented on ,,when and where required basis [16]. In order to justify the business case to apply a new modular software application for virtual commissioning of the engine assembly machines, a review of current prevalent practices at Ford is presented briefly. The current reconfiguration approach is largely based on the skill and knowledge of engineers rather than the actual process involved. Whenever there is any change in the product it is then essentially engineer's responsibility to examine the needs of the reconfigured system to support the new product [16].

Therefore there is a need to introduce decision support for assembly line design, build and/or reconfiguration activity. It is intended that a comparison of the existing practices among engineers against the proposed future state will outline the benefits of using CCE modular tool and provide a roadmap to increased efficiency and streamlined information exchange. Data 
collection activity has primarily been based around structured interviews and mapping highlevel information flows over the course of a program and involved Ford engineers, external suppliers and information collected through other team members working on the project.

The principal focus of the study looked at common machine mechanisms on the engine assembly lines. To support the activities of engineering partners, largely ad hoc integration methods and mechanisms are currently employed. A mechanism can be either physical (i.e. actuators, lifts, clamps), logical (i.e. RF data readers, position interrogation sensors) or structural (i.e. conveyance, fixturing). Mechanism decomposition can be viewed from three levels, firstly functionally: what is the physical operation to be performed by the mechanism (i.e. translation, join, test etc), secondly process: what process steps does the mechanism perform to achieve the function (i.e. grasp, rotate $180^{\circ}$, etc) and finally detail: considers mechanisms at a low level looking at the control logic, geometric, hydraulic, pneumatic, electrical requirements that combine to fulfil the mechanism function as shown in figure 12.

\begin{tabular}{|c|c|l|c|}
\hline \# ID & $\begin{array}{c}\text { Mechanism } \\
\text { Name }\end{array}$ & $\begin{array}{c}\text { Function } \\
\text { Y Axis } \\
\text { Translation }\end{array}$ & $\begin{array}{l}\text { Lifts both the part (20mm) by clamping the } \\
\text { mounting plate to achieve a clamped in process } \\
\text { part that is zeroed in 3-D space. }\end{array}$ \\
\hline 2 & $\begin{array}{c}\text { Y Axis } \\
\text { Translation }\end{array}$ & $\begin{array}{l}\text { Lifts both the part (550mm max) and mounting } \\
\text { to achieve a clamped in process part that is } \\
\text { zeroed in 3-D space. }\end{array}$ \\
\hline Rotate Part & $\begin{array}{l}\text { Rotate at raised position requires allows a part } \\
\text { to be turned +/- 225 max before being returned } \\
\text { to the pallet base. }\end{array}$ \\
\hline Clamping Unit & $\begin{array}{l}\text { Clamps the part mounting in order to allow } \\
\text { either a translation operation or to allow } \\
\text { gauging or tooling to engage on the part } \\
\text { knowing that it is zeroed in 3D space. }\end{array}$ \\
\hline
\end{tabular}

Figure 12. Defining modular machine mechanisms

The rationale to the stated decomposition is that an assembly machine is a combination of several independent functional units which can be re-designed/re-arranged to form new machines or re-configured to accommodate changes in the product i.e. engine.

This research aims to gain a detailed understanding of data producers, data consumers and format/flow of the data within Ford especially for defining and using Bill of Process (BoP) for engine assembly lines. The purpose of this case study is to focus on the real industrial challenge of rapid constraint evaluation for designing and/or reconfiguration of Powertrain assembly lines to cater for a new/changed product. By introducing the standardised (i) library components of machines, (ii) data structures in CCE tool and later (iii) ontological knowledge management techniques for applications / database integration and re-configuration management in the target domain at Ford, a revised and improved business case is presented here. This research aims to gain a detailed understanding of data communication and so that a consistent specification of the CCE tool can be formulated as well as integration with the legacy applications could be made a reality especially for defining and using BoP for engine assembly lines. 
A business case is presented where a process engineer needs to commission a new engine assembly line. The engineer will apply his own and other engineers' knowledge to evaluate changes and find appropriate components / modules for the changed machine/line and then pass on the data to machine tool builders with suggestions. For the suggested changed system, the engineer will virtually build new or re-configure existing machines at Ford with CCE tool using pre-built machine components, modules and mechanisms with the available option of a complete BoP in CCE tool and pass on the data with more confidence and without changes and amendments cycle between Ford and vendors as shown in figure 13.

As-Is
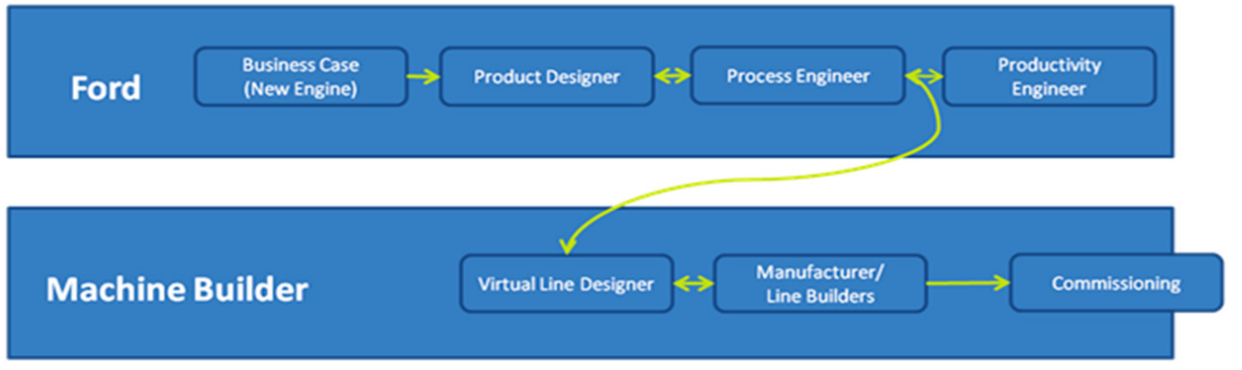

To-Be

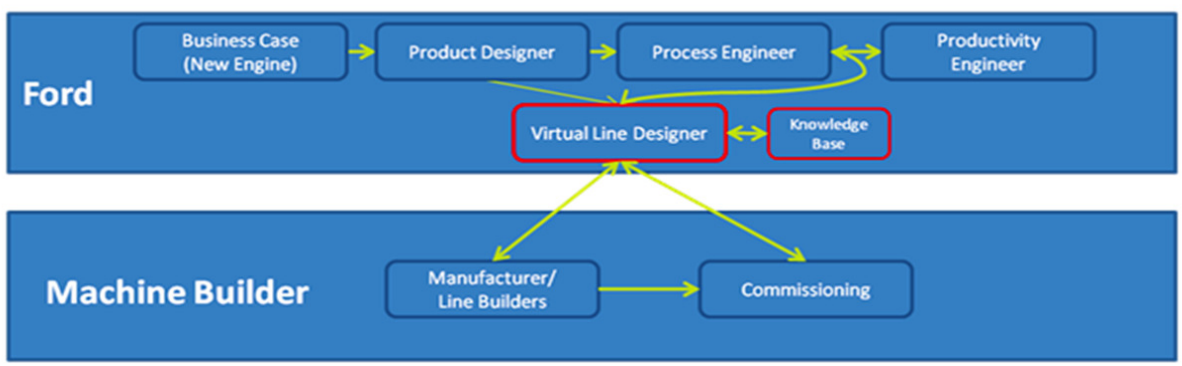

Figure 13. ‘As-is' vs. ‘To-be' business process for powertrain assembly line

\section{Principal Findings}

\subsection{Data Structures for Generic BoP}

A fundamental enabler for the delivery of a revised engineering process of building engine assembly machines is standardised data structures. For any changed product, approximately $70 \%$ of the assembly line machines remain the same, only 30\% (approx) of machines / modules are required to be built for the changed product. Therefore, knowledge reuse and applications' integration becomes a pivotal task in increasing efficiency of the process. Definitions, characteristics and classification of a generic $\mathrm{BoP}$ would help process engineers to quickly build the machines in virtual environment and test and de-bug if required. The operational behaviour of the CCE application is principally agreed upon between Loughborough University and Ford Company but the data input/output characteristics need to be defined and documented. Prospective system will have a resource library of assembly machines and modules in hierarchical form and Ford engineers would be able to make the full use of previous data to virtually build the machines quickly and pass this information on to its vendors with more confidence. 
CCE is a software application developed at Loughborough University which aids in simulating the virtual commissioning of the assembly machine systems in general and automotive engine assembly line in particular. The CCE tool development was initiated based on the requirements of the automotive industry in terms of large-scale complex automation system design and commissioning. CCE is a lightweight $3 \mathrm{D}$ simulation tool to visualise, test, debug and validate the machine behaviour in a virtual environment. The output of CCE can be expressed in terms of $\mathrm{xml}$ format which is an open data format which renders future integration capabilities with other legacy systems.

The CCE tool development is driven by the concepts of a "modular-based system" architecture which seeks to enable re-usability and re-configurability of basic modelling constructs. The concept of a 'module' is defined as a re-usable and re-configurable resource. In modular architecture any one component / module can be replaced, removed or added without affecting the rest of the system.

A systematic taxonomical structure and associated properties for designing / reconfiguring machine tools for automotive assembly lines is presented here. These operations are decomposed into a set of functions that the machine must perform and the functions are mapped to machine modules. Therefore 'assembly operation' in PD corresponds to 'system' in CCE, 'assembly process' to 'module' and 'assembly task' to 'component'.

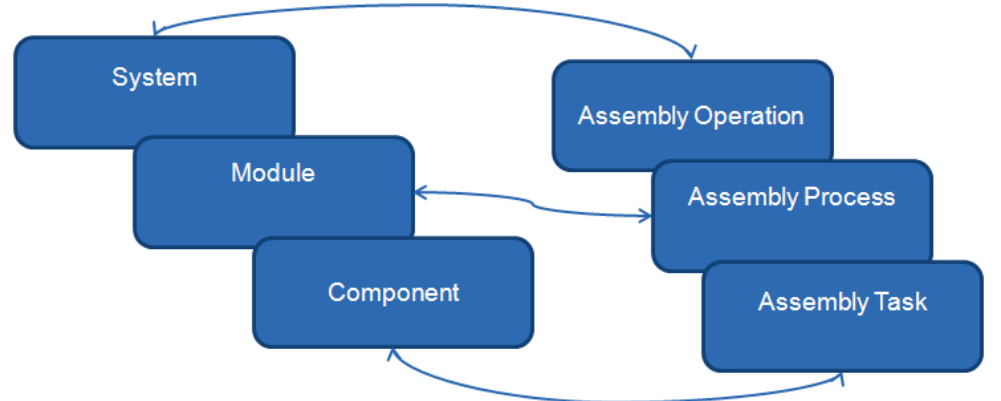

Figure 14. Decomposition in CCE tool consistent to the assembly BoP

The reason the CCE has three main tabs is that it corresponds to the decomposition of assembly operations in real industrial scenario as shown in figure 14. Assembly Operation may be considered as a combination of Assembly Process (sub tasks) which in turn is combination of Assembly Tasks (unit assembly act). To understand and define properties and structure, it is necessary to envisage the application scenarios of the CCE application tool. There are several ways in which this application can be used. To define a consistent structure with other applications, following categories of components, modules and systems are implemented:

1. End User - Ford - Program-based

\{Predecessor/Successor - Constructional \& Operational\}

2. Machine Builder - Krause - vendor based

3. Assembly Operation - Generic BoP - Assembly Task based

\section{Miscellaneous}

Every operation has to be related with the product. For a particular operation X, we need to have product level at this point. An important category i.e. Product Level Assembly corresponding to systems can be associated with each category. Product is defined as a component with no control / state behaviour. The corresponding level of product can be made visible from a 
complete assembled product and the higher levels can be made invisible. This is exactly in compatible with the TC structure.

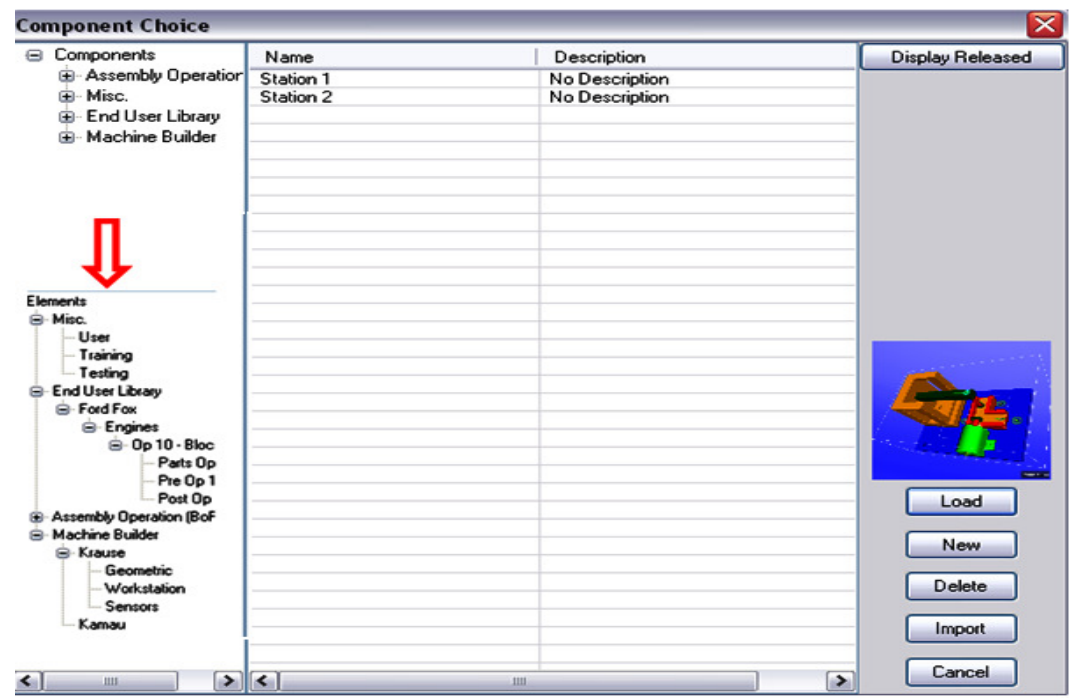

Figure 15. Component choice categories in CCE tool

\section{Conclusion ANd Future ACTIVITIES}

The ontological representations are engineering artefacts, conflation of related entities in a domain model and an intersection of cognitive science, object oriented concept, lingistics, philosophy and episetomology. To explicitly define domain concepts at Ford, ontologies are used for data capturing and representation thus integrating PLM system efficiently into common factory floor information platform. Business Process Management (BPM) provides real-time, cross-functional visibility into operational processes and a common understanding of activities for all participants. With BPM, the execution of many process tasks can be automated that may have previously been handled manually by utilising the existing services or adding the new ones. For tasks that still require manual handling, BPM will coordinate the workflow and direct action by notifying people and presenting them the information they need to perform their work. Successful business now requires seamless integration of processes and the instant exchange of information on a planetary scale. BPM suite supports service oriented architecture like ontology supports SOA. It is aimed in future to integrate BPM with existing applications through semantics in the form of ontology, as shown in figure 16, to enable the business process such as reconfiguration of the line to be linked directly to the appropriate domain knowledge.

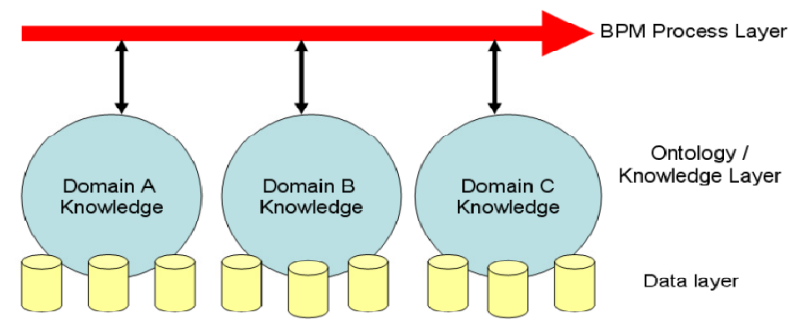

Fig. 16: Business processes routed in domain knowledge

Starting from already completed business process modelling work, the validated and approved models of Ford engine assembly business processes are available. CCE tool is covering only manufacturing engineering domain (DP5) and machine builders domain (DP6) at Ford. Other 
International Journal of Advanced Information Technology (IJAIT) Vol. 1, No. 3, June 2011

areas are still independent silos where the integration lacks amongst themselves as well as the information handling is dependent upon software/databases specific to their needs. There exists a need to integrate this process to share information among all the stake holders for new design or reconfiguration of the line. This will be applicable at Dunton research facility of Ford.

As manufacturing trends impose increasing demands for autonomous collaboration, reconfigurability and heterogeneous system integration, the current control architectures become inflexible and inefficient. The use of ontologies and semantic web services as interaction model is set to overcome current interoperability barriers. Through interpretation of explicit semantics and inference, machines can learn about the manufacturing skills and conversational skills of other machines to a sufficient degree to enable automatic discovery, classification, composition and orchestration of manufacturing equipment. Semantic web services can be incrementally implemented into existing control architecture in order to enhance autonomy, interoperability and rapid re-configurability. The goal of modern assembly planning systems is to create activity sequences that are not only feasible but also optimised according to one or more parameters. Ontologies and semantic web services can help achieve the purpose through management of knowledge and information especially for integration of PPR domains.

\section{ACKNOWLEDGEMENTS}

The authors gratefully acknowledge the support of UK EPSRC and Loughborough University's IMCRC through Business Driven Automation (BDA) project. We would like to thank all the project participants and engineers who have contributed in this research from Loughborough University and participant companies especially Ford Motor Company, UK.

\section{REFERENCES}

[1] Nof S.Y., G. Morel, L. Monostori, A. Molian, F. Flip, From plant and logistics control to multienterprise Collaboration. 2005, Status report prepared by the IFAC Coordination Committee on Manufacturing Systems.

[2] Vernadat F.B., Enterprise modelling and integration: Principles and applications, 1996, ISBN 0412605503, Chapman and Hall.

[3] Theodore J. Williams, Hong Li, PERA AND GERAM-- A Vision of Enterprise Integration Considerations, A holistic perspective as shown by the Purdue Enterprise Reference Architecture, IFIP International Federation for Information Processing, 2005, Volume 183/2005, 249-267,

[4] Sebastian K. Fixson, 2006. Modularity and Commonality Research: Past developments and Future Opportunities, Sloan School of Management, MIT Sloan Working Paper 4629-06.

[5] Erixon, G. and Östgren, B. 1993, Synthesis and evaluation tool for modular designs. N.F.M Roozenburg (ed.), Proceedings of the 9th International Conference on Engineering Design 93, The Hague. 898-905.

[6] Brown J., K Rathmill, S.P. Sethi and K.E. Stecke, Classification of flexible manufacturing systems, The FMS magazine, April 1984, pp 114 - 117.

[7] Chryssolouris G, Manufacturing Systems Theory and Practice, Springer - Verlag, Newyork, (1992), pp 16-23. 
International Journal of Advanced Information Technology (IJAIT) Vol. 1, No. 3, June 2011

[8] Elkins D.A., N. Huang, J.M. Aldin, Agile manufacturing systems in the automotive industry, 2004, International journal of production economics, vol. 91, pp 201-214.

[9] Mehrabi M.G., A. G. Ulsoy, y. Koren. Reconfigurable manufacturing systems: Key to future manufacturing. Journal of Intelligent Manufacturing, Volume 11, Number 4, August 2000.

Kluwer Academic Publishers. Pp. 403-419.

[10] Leitao P., J Barata, L. Camarinha Matos, R. Boissier, Trends in agile and cooperative manufacturing, 2001, Proceedings of low cost production.

[11] John A. Slotwinski, Robert B. Tilove, Smart Assembly, Industry needs and challenges. National Institute of Standards and Technology. http://smartassembly.wikispaces.com/

[12] Hopgood A.A. 2001, Intelligent systems for engineers and scientists, CRC Press, $2^{\text {nd }}$ edition, ed. 2000, Boca Raton, Florida, USA

[13] Sriram R.D. 1997, Intelligent systems for engineering, A knowledge based approach, SpringerVerlag Ltd., London, Great Britain.

[14] M. Kifer, G. Lausen, and J. Wu. Logical Foundations of Object-Oriented and Frame-Based Languages. JACM, 42(4):741-843, 1995.

[15] BDA project at Loughborough University: http://www.lboro.ac.uk/departments/mm/research/manufacturing-systems/dsg/bda/index.htm

[16] M.B. Raza, T. Kirkham, R. Harrison, Knowledge Based Flexible and Integrated PLM System at Ford, Journal of Information \& Systems Management, Volume 1, Number 1, March 2011, Pages: 8-16.

\section{Authors}

Muhammad Baqar Raza is a $\mathrm{PhD}$ student at Loughborough University, UK. His $\mathrm{PhD}$ is about ontological knowledge management techniques in manufacturing and automation systems. He did his BSc in Mechanical Engineering from UET, Pakistan, obtained his MSc degree in Product Engineering from Cranfield University, UK. He worked for more than 08 years in various industrial domains in different capacities, including shop-floor supervisor, quality engineer, production engineer and research collaborator. His research area includes manufacturing knowledge management, service oriented computing, quality control and quality management systems in automotive and aerospace sectors.

Prof. Dr. Robert Harrison is a Professor at Wolfson School of Mechanical and Manufacturing Engineering, Loughborough University, UK. He is a visiting Research Fellow at the National University of Singapore, $\mathrm{PhD}$ in distributed machine control systems from Loughborough University. He is Research Fellow at Ford Motor Company, UK from 1996 to date. His research areas are diversified and mainly focused on systems modelling, integration, control, modular and reconfigurable manufacturing and automation systems especially creating globally distributed virtual environments to underpin the lifecycle engineering of component-based machines and process control systems. 